\title{
UV PROPERTIES OF EARLY-TYPE GALAXIES IN THE VIRGO CLUSTER
}

\author{
A. Boselli, ${ }^{1}$ L. Cortese, ${ }^{1}$ J. M. Deharveng, ${ }^{1}$ G. Gavazzi,${ }^{2}$ K. S. Yi,${ }^{3}$ A. Gil de Paz, ${ }^{4}$ M. Seibert, ${ }^{5}$ S. Boissier, ${ }^{4}$ \\ J. Donas, ${ }^{1}$ Y.-W. Lee, ${ }^{6}$ B. F. Madore, ${ }^{4,7}$ D. C. Martin, ${ }^{5}$ R. M. Rich, ${ }^{8}$ And Y.-J. Sohn ${ }^{6}$ \\ Received 2005 April 25; accepted 2005 July 6; published 2005 July 27
}

\begin{abstract}
We study the UV properties of a volume-limited sample of early-type galaxies in the Virgo Cluster combining new GALEX far-ultraviolet $(1530 \AA)$ and near-ultraviolet $(2310 \AA)$ data with spectrophotometric data available at other wavelengths. The sample includes 264 elliptical, lenticular, and dwarf galaxies spanning a large range in luminosity $\left(M_{B} \leq-15\right)$. While the NUV to optical or near-IR color-magnitude relations (CMRs) are similar to those observed at optical wavelengths, with a monotonic reddening of the color index with increasing luminosity, the $(\mathrm{FUV}-V)$ and $(\mathrm{FUV}-H) \mathrm{CMRs}$ show a discontinuity between massive and dwarf objects. An even more pronounced dichotomy is observed in the (FUV - NUV) CMR. For elliptical galaxies, the (FUV - NUV) color becomes bluer with increasing luminosity and with increasing reddening of the optical or near-IR color indices. For the dwarfs, the opposite trend is observed. These observational evidences are consistent with the idea that the UV emission is dominated by hot, evolved stars in giant systems, while in dwarf ellipticals residual star formation activity is more common.
\end{abstract}

Subject headings: galaxies: clusters: individual (Virgo) — galaxies: elliptical and lenticular, cD galaxies: evolution — ultraviolet: galaxies

\section{INTRODUCTION}

The excess ultraviolet radiation from early-type galaxies arises from hot stars in late stages of stellar evolution (O'Connell 1999). Whether the so-called UV upturn depends on the detailed galaxy morphology (ellipticals vs. lenticulars) and, among ellipticals, on luminosity (dE's vs. giant E's) is yet unknown. It would not be surprising if the UV properties of dwarf elliptical galaxies would differ from those of giants, given that other structural (Gavazzi et al. 2005) and kinematic (Van Zee et al. 2004) properties depend on luminosity, due to different star formation histories (single episodic vs. burst; Ferguson \& Binggeli 1994; Grebel 1999).

Due to morphological segregation (Whitmore et al. 1993), nearby clusters are the ideal targets for assembling complete, volume-limited samples of early-type objects. As part of a study aimed at analyzing the environmental dependence of galaxy evolution, we observed large portions of the Virgo Cluster with the Galaxy Evolution Explorer (GALEX; Boselli et al. 2005). Owing to the superior quality of the photographic material obtained by Sandage and collaborators, an extremely accurate and homogeneous morphological classification exists for Virgo galaxies, down to $m_{B} \leq 18 \mathrm{mag}\left(M_{B} \leq-13\right.$ assuming a distance of $17 \mathrm{Mpc}$ ), allowing a detailed discrimination among different subclasses of early-type galaxies (ellipticals, lenticulars, dwarfs) and an exclusion of quiescent spirals. Furthermore, a wealth of

\footnotetext{
${ }^{1}$ Laboratoire d'Astrophysique de Marseille, BP8, Traverse du Siphon, F13376 Marseille, France.

${ }^{2}$ Università degli Studi di Milano-Bicocca, Piazza della Scienza 3, 20126 Milano, Italy.

${ }^{3}$ Department of Astrophysics, Oxford University, Oxford OX1 3RH, UK.

${ }^{4}$ Observatories of the Carnegie Institution of Washington, 813 Santa Barbara Street, Pasadena, CA 91101.

${ }^{5}$ California Institute of Technology, Mail Code 405-47, 1200 East California Boulevard, Pasadena, CA 91125.

${ }^{6}$ Center for Space Astrophysics, Yonsei University, Seoul 120-749, South Korea.

${ }^{7}$ NASA/IPAC Extragalactic Database, California Institute of Technology, Mail Code 100-22, 770 South Wilson Avenue, Pasadena, CA 91125.

${ }^{8}$ Department of Physics and Astronomy, University of California, Los Angeles, CA 90095 .
}

ancillary data for many Virgo members, covering a large portion of the electromagnetic spectrum from the visible to the infrared, is available from the GOLDMine database (Gavazzi et al. 2003).

\section{DATA}

The analysis presented in this work is based on an optically selected sample of early-type galaxies including giant and dwarf systems (E, S0, S0a, dE, and dS0) extracted from the Virgo Cluster catalog of Binggeli et al. (1985), which is complete to $m_{B} \leq 18 \mathrm{mag}\left(M_{B} \leq-13\right)$. The Virgo Cluster region was observed in spring 2004 as part of the All Imaging Survey (AIS) and of the Nearby Galaxy Survey (NGS) carried out by GALEX in two UV bands: the far-ultraviolet (FUV; $\lambda_{\text {eff }}=$ $1530 \AA, \Delta \lambda=400 \AA$ ) and the near-ultraviolet (NUV; $\lambda_{\text {eff }}=2310 \AA, \Delta \lambda=1000 \AA$ ), covering 427 objects. Details of the GALEX instrument and data characteristics can be found in Martin et al. (2005) and Morrissey et al. (2005).

The present sample includes all Virgo Cluster early-type systems detected in the NUV GALEX band (264 objects, 194 from the NGS); of these, 126 (of which 74 are from the NGS) have been also detected in the FUV. The resulting sample is thus ideal for the proposed analysis, as it provides us with the first large volume-limited sample of elliptical, lenticular, and dwarf galaxies spanning 4 dex in luminosity with homogeneous data. Whenever available, we extracted fluxes from the deep NGS images, obtained with an average integration time of $\sim 1500 \mathrm{~s}$, complete to $m_{\mathrm{AB}} \sim 21.5$ in the NUV and FUV. Elsewhere UV fluxes have been extracted from the less deep AIS images $\left(\sim 70 \mathrm{deg}^{2}\right)$, obtained with an average integration time of $\sim 100 \mathrm{~s}$, complete to $m_{\mathrm{AB}} \sim 20$ in both the FUV and NUV bands. The resulting sample, although not complete in both UV bands, includes giants and dwarf systems: at a limiting magnitude of $M_{B} \leq-15,71 \%$ of the observed galaxies have been detected in the NUV, $46 \%$ in the FUV.

All UV images come from the GALEX IR1.0 release. UV fluxes were obtained by integrating GALEX images within elliptical annuli of increasing diameter up to the optical $B$-band $25 \mathrm{mag} \operatorname{arcsec}^{-2}$ isophotal radii consistently with the optical and near-IR images. Independent measurements of the same 

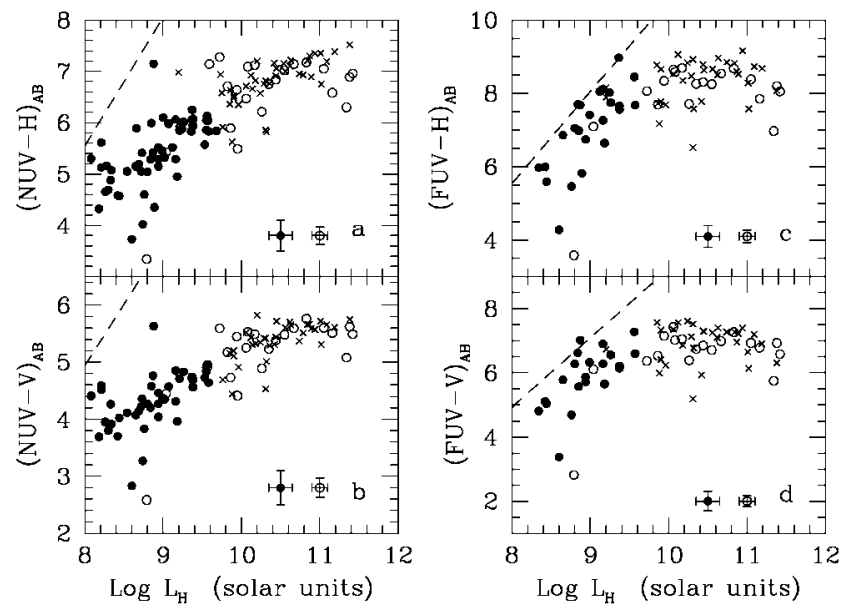

FIG. 1.-NUV (left column) and FUV (right column) to optical and nearIR color-magnitude relations. Colors are in the AB magnitude system. Open circles are for ellipticals, filled circles are for dwarfs, and crosses are for lenticulars (SO-SOa). Galaxies redder than the dashed line are undetectable by the present survey (at the NGS limit). Largest $1 \sigma$ errors for luminous and dwarf systems are given.

galaxies obtained in different exposures give consistent photometric results within $10 \%$ in the NUV and $15 \%$ in the FUV in the AIS and about a factor of 2 better for bright (NUV $\leq$ 16) galaxies. The statistical uncertainty in the UV photometry is on average a factor of $\sim 2$ better in the NGS than in the AIS, especially for fainter objects.

UV data have been combined with multifrequency data taken from the GOLDMine database ${ }^{9}$ (Gavazzi et al. 2003). These are $B$ and $V$ imaging data, mostly from Gavazzi et al. (2005) and Boselli et al. (2003), and near-IR $H$ imaging from Gavazzi et al. (2000, 2001). Optical and near-IR data have on average a photometric precision of $\sim 10 \%$. Spectroscopic metallicity index $\mathrm{Mg}_{2}$ and velocity dispersion data come from GOLDMine or from Golev \& Prugniel (1998) and Bernardi et al. (2002). Unless specified, we adopt the homogeneous morphological classification of Binggeli et al. (1985) based on high-quality photographic material.

Galaxies analyzed in this work are all bona fide Virgo Cluster members: given the three-dimensional structure of the cluster, distances have been assigned following the subcluster membership criteria of Gavazzi et al. (1999). Owing to the high Galactic latitude of Virgo, no Galactic extinction correction was applied $\left(A_{B} \leq 0.05\right)$.

\section{UV PROPERTIES OF EARLY-TYPE GALAXIES}

Despite the complex three-dimensional structure of Virgo (Gavazzi et al. 1999), the uncertainty on the distance (hence on the luminosity) of the target galaxies does not constitute a major source of dispersion in the determination of the colormagnitude relation (CMR). Figure 1 shows various UV to optical and near-IR CMRs. Similar results are obtained if, instead of the mass-tracer $H$-band luminosity (Zibetti et al. 2002), we use the $B$-band absolute magnitude.

The NUV to optical (Fig. 1b) and near-IR (Fig. 1a) CMRs are well defined and are similar to optical or near-IR CMRs, with brighter galaxies having redder colors, independent of their morphological type: the color index (NUV $-V$ ) increases by $\sim 2$ mag from dwarfs $\left(L_{H} \sim 10^{8} L_{H, \odot}\right)$ to giants $\left(L_{H} \sim\right.$

${ }^{9}$ See http://goldmine.mib.infn.it.
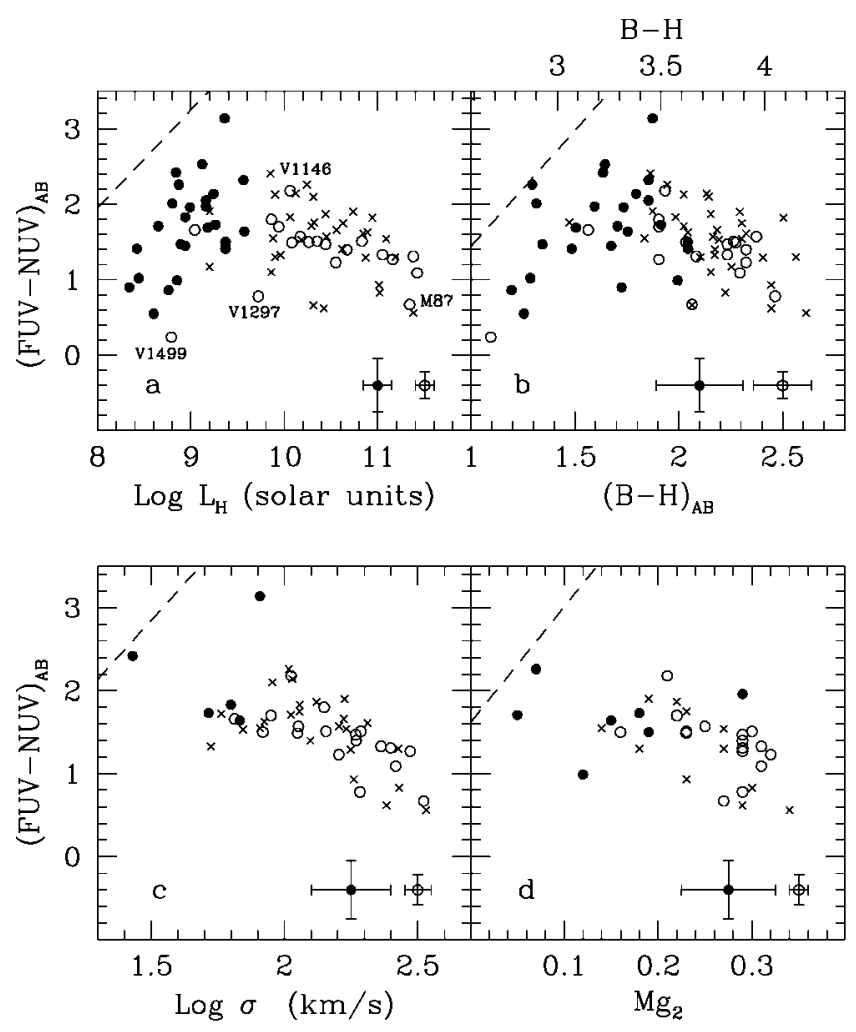

FIG. 2.-Relationship between the UV color index (FUV - NUV) and (a) the total $H$-band luminosity, (b) the $(B-H)$ color index, (c) the logarithm of the central velocity dispersion, and $(d)$ the $\mathrm{Mg}_{2}$ index. Symbols are as in Fig. 1. Labeled points indicate objects having unusual radio or optical properties (see § 3 ).

$10^{11.5} L_{H, \odot}$ ), while (NUV $-H$ ) changes by $\sim 3$ mag. A weak flattening of the relation appears for $L_{H} \geq 10^{10} L_{H, \odot}$. This behavior confirms the one reported by Ferguson (1994) in the $(B-V)$ versus $M_{B}$ CMR.

On the contrary, the FUV to optical (Fig. 1d) and near-IR (Fig. 1c) CMRs differ systematically for dwarfs and giant systems: galaxies brighter than $L_{H} \sim 10^{9.5} L_{H, \odot}$ have similar red colors, while for $L_{H} \leq 10^{9.5} L_{H} \odot$ colors become progressively bluer. Even if this trend can be due to a selection effect (reddest dwarfs being undetectable in the FUV), it is indisputable that there exists a significant population of dE's with bluer colors than E's and S0's.

A similar pattern (systematic differences between massive and dwarf systems and between NUV and FUV to optical or near-IR color indices) is obtained using color-color diagrams (not shown).

The dichotomy between giants and dwarfs is even more apparent in the UV color index (FUV - NUV) (see Fig. 2). The (FUV - NUV) becomes redder with increasing luminosity for dwarf ellipticals while, on the contrary, it becomes bluer for giant ellipticals (Fig. 2a). The blueing relation is tight among ellipticals (see Table 1) and barely observed in lenticulars because of their higher dispersion. ${ }^{10} \mathrm{~A}$ similar behavior between ellipticals

\footnotetext{
${ }^{10}$ The scatter in the blueing relation among ellipticals decreases significantly (from 0.31 to 0.10 ) if we exclude the misclassified poststarburst dwarf VCC 1499 (Gavazzi et al. 2001; Deharveng et al. 2002), the radio galaxy M87, VCC 1297 (the highest surface brightness galaxy in the sample of Gavazzi et al. 2005), and VCC 1146. Besides its extremely high surface brightness, making VCC 1297 a nonstandard object, we do not have any evidence indicating a peculiar star formation history or present nuclear activity in VCC 1297 and VCC 1146 that could justify their exclusion.
} 
TABLE 1

Main Relations for Early-Type Galaxies

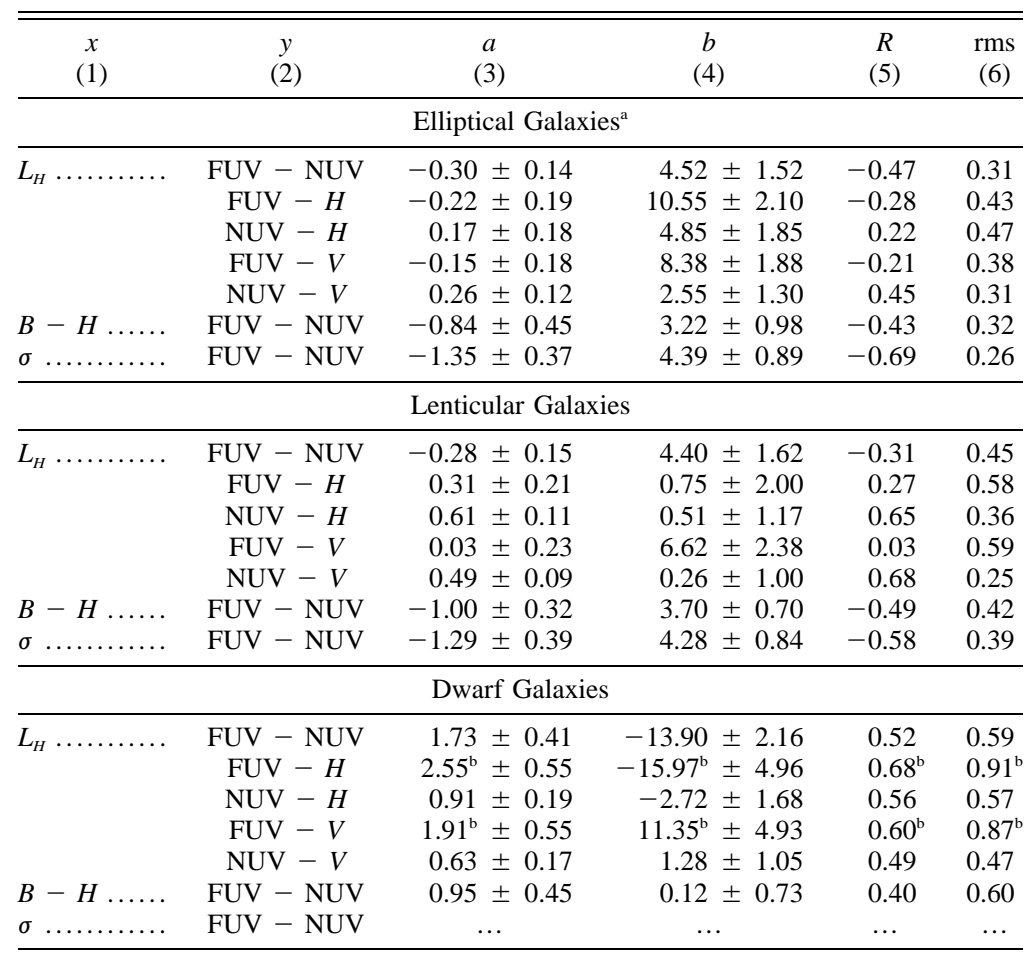

Note. -Cols. (1) and (2): $x$ and $y$ variables. Cols. (3) and (4): Slope $a$ and intercept $b$ of the bisector linear fit with weighted variables. Col. (5): Pearson correlation coefficient. Col. (6): Mean dispersion around the best fit.

${ }^{\text {a }}$ Excluding VCC 1499.

${ }^{\mathrm{b}}$ Uncertain values because of the UV detection limit.

and lenticulars is observed in the (FUV - NUV) color relation (Fig. 2b): this mixed giant population becomes bluer in the UV with increasing reddening in the $(B-H)$ color index. The behavior of dwarf ellipticals is different: although with a huge dispersion, the (FUV - NUV) color index reddens as the $(B-H),(B-V)$, and (FUV $-V)$ indices (the two last not shown). The dichotomy between dwarf and giant systems cannot be observed in the run of (FUV - NUV) versus central velocity dispersion (which is directly related to the system total dynamical mass; Fig. 2c) nor as a function of the metallicity-sensitive (Poggianti et al. 2001) $\mathrm{Mg}_{2}$ Lick index (Fig. 2d), because these two parameters are not available for dwarfs. In ellipticals and lenticulars, the UV color index (FUV - NUV) depends on both the metallicity index $\mathrm{Mg}_{2}$ and $\sigma$ in a way opposite to the behavior at optical wavelengths, where galaxies are redder when having higher $\mathrm{Mg}_{2}$ and velocity dispersions.

\section{DISCUSSION AND CONCLUSION}

For the first time, the UV properties of early-type galaxies have been studied down to $M_{B} \sim-15 \mathrm{mag}$. The comparison with previous studies is thus limited to the brightest objects. Our CMR can be compared with the one obtained by Yi et al. (2005) based on a complete sample of bright early-type objects $\left(M_{r} \leq\right.$ $-20 \mathrm{mag}$ ) extracted from the Sloan Digital Sky Survey (SDSS) by Bernardi et al. (2003). The CMR presented by Yi et al. (2005; NUV $-r$ vs. $\left.M_{r}\right)$ shows a significantly larger dispersion $(\sigma \geq$ $1.5 \mathrm{mag}$ ) than the one found in Virgo (see Table 1). As discussed in Yi et al. (2005), the large dispersion in their CMR can be ascribed to galaxies with a mild or residual star formation activity included in the Bernardi et al. (2003) sample. If restricted to the "UV-weak" sample, the dispersion in the Yi et al. relation drops to $0.58 \mathrm{mag}$, i.e., still larger than the one seen in the Virgo Cluster in the same luminosity range. Despite possible larger distance uncertainties in the SDSS, the difference in the scatter between our and the Yi et al. (2005) CMR might arise from the classification in the SDSS that uses concentration indices and luminosity profiles in discriminating hot from rotating systems. It is in fact conceivable that the larger dispersion in the CMR of UVweak galaxies of Yi et al. (2005) comes from the contamination of quiescent, bulge-dominated Sa spiral disks that have structural (concentration indices and light profiles) or population properties (colors and spectra) similar to ellipticals and lenticulars (Scodeggio et al. 2002; Gavazzi et al. 2002).

The monotonic increase of the (NUV $-V$ ) and (NUV $-H$ ) colors with luminosity, similar to the one observed in the visible bands by Ferguson (1994) and Ferguson \& Binggeli (1994), strongly suggests that both in dwarfs and giant systems the NUV $2310 \AA$ flux is dominated by the same stellar population emitting at longer wavelengths. The plateau and the higher dispersion observed in the FUV CMRs confirm that the UV upturn is observable only in the FUV GALEX band at $1530 \AA$, as already remarked by Dorman et al. (2003) and Rich et al. (2005).

The mild trend between the (FUV - NUV) color and the metallicity-sensitive $\mathrm{Mg}_{2}$ index, as seen in the present data set, is predicted by models (Bressan et al. 1994; Yi et al. 1998). It is also in qualitative agreement with the early International Ultraviolet Explorer (IUE) result of Burstein et al. (1988). However, it should be noted that the Burstein data set is systematically different from the GALEX data set: Burstein et al. used a different color index, $(1550 \AA-V)$, which was computed inside a fixed $I U E$ aperture. On the other hand, the trend noted here contrasts with the analysis of Rich et al. (2005), 
who recently reported the lack of a significant correlation between the (FUV $-r$ ) color index and $\mathrm{Mg}_{2}$, and the velocity dispersion $\sigma$. They examined a large sample of SDSS earlytype galaxies, also observed by GALEX. We can only speculate that the lack of correlation in the data set may be the result of a relatively restricted dynamic range in the variables available to those authors at that time: only $2.1-2.4 \mathrm{~km} \mathrm{~s}^{-1}$ in $\log \sigma$ and $0.18-0.30$ in $\mathrm{Mg}_{2}$. Clearly the issue is not decided and would benefit from targeted new observations.

The newest result of the present Letter, shown in Figure 2, addresses the question raised by O'Connell (1999) concerning the dependence of the UV properties on galaxy morphology. We have shown that a dichotomy exists between giant and dwarf ellipticals and, to a lesser extent, between ellipticals and lenticulars. The blueing of the UV color index with luminosity, metallicity, and velocity dispersion indicates that the UV upturn is more important in massive, metal-rich systems.

The accurate morphological classification in our sample allows us to discriminate between $\mathrm{E}$ and S0s. The higher dispersion in the $(\mathrm{FUV}-\mathrm{NUV})$ versus $L_{H}$ relation observed for the lenticulars compared to the extremely tight one for ellipticals (see Table 1) bears witness to recent, minor episodes of star formations combined with an old stellar population, as determined also from kinematic and spectroscopic observations (Dressler \& Sandage 1983; Neistein et al. 1999; Hinz et al. 2003). We have shown that the UV properties of ellipticals are different than those of lenticulars, suggesting a different evolution.

The opposite behavior (reddening of the UV color index with luminosity) of dwarfs with respect to giants, similar to that ob- served for spirals, indicates that the UV spectra of low-luminosity objects are shaped by the contribution of young stars and thus are more sensitive to the galaxy's star formation history than to the metallicity. Spectroscopy was recently obtained (unpublished) for the seven bluest dE's [(FUV - NUV) $\leq 1.4 \mathrm{mag}$ ] in our sample. Three out of seven show Balmer emission lines and another three strong $\mathrm{H} \delta$ in absorption $(\mathrm{H} \delta \mathrm{EW} \geq 5 \AA$ ) witnessing a present or recent star formation activity. This implies that the stellar population of dwarfs has been formed in discrete and relatively recent episodes, as observed in other nearby objects (Grebel 1999).

More evidences are building up that mass drives the star formation history in hot systems (Trager et al. 2000; Gavazzi et al. 2002; Caldwell et al. 2003; Poggianti 2004) as in rotating ones (Gavazzi et al. 1996, 2002; Boselli et al. 2001) and that the stellar population of massive ellipticals is on average older than that of dwarfs.

We thank an unknown referee for his/her criticism. GALEX (Galaxy Evolution Explorer) is a NASA Small Explorer, launched in 2003 April. We gratefully acknowledge NASA's support for construction, operation, and science analysis for the GALEX mission, developed in cooperation with the Centre National d'Etudes Spatiales of France and the Korean Ministry of Science and Technology. The authors would like to take this opportunity to thank the members of the GALEX SODA team for their valiant efforts in the timely reduction of the complex observational data set covering the full expanse of the Virgo Cluster.

\section{REFERENCES}

Bernardi, M., et al. 2002, AJ, 123, 2990 2003, AJ, 125, 1817

Binggeli, B., Sandage, A., \& Tammann, G. 1985, AJ, 90, 1681

Boselli, A., Gavazzi, G., Donas, J., \& Scodeggio, M. 2001, AJ, 121, 753

Boselli, A., Gavazzi, G., \& Sanvito, G. 2003, A\&A, 402, 37

Boselli, A., et al. 2005, ApJ, 623, L13

Bressan, A., Chiosi, C., \& Fagotto, F. 1994, ApJS, 94, 63

Burstein, D., Bertola, F., Buson, L., Faber, S., \& Lauer, T. 1988, ApJ, 328, 440

Caldwell, N., Rose, J. A., \& Concannon, K. D. 2003, AJ, 125, 2891

Deharveng, J.-M., Boselli, A., \& Donas, J. 2002, A\&A, 393, 843

Dorman, B., O’Connell, R. W., \& Rood, R. T. 2003, ApJ, 591, 878

Dressler, A., \& Sandage, A. 1983, ApJ, 265, 664

Ferguson, H. 1994, in Dwarf Galaxies, ed. G. Meylan \& P. Prugniel (Garching: ESO), 475

Ferguson, H., \& Binggeli, B. 1994, A\&A Rev., 6, 67

Gavazzi, G., Bonfanti, C., Sanvito, C., Boselli, A., \& Scodeggio, M. 2002, ApJ, 576, 135

Gavazzi, G., Boselli, A., Donati, A., Franzetti, P., \& Scodeggio, M. 2003, A\&A, 400, 451

Gavazzi, G., Boselli, A., Scodeggio, M., Pierini, D., \& Belsole, E. 1999, MNRAS, 304, 595

Gavazzi, G., Donati, A., Cucciati, O., Sabatini, S., Boselli, A., Davies, J., \& Zibetti, S. 2005, A\&A, 430, 411

Gavazzi, G., Franzetti, P., Scodeggio, M., Boselli, A., Pierini, D., Baffa, C., Lisi, F., \& Hunt, L. K. 2000, A\&AS, 142, 65
Gavazzi, G., Pierini, D., \& Boselli, A. 1996, A\&A, 312, 397

Gavazzi, G., Zibetti, S., Boselli, A., Franzetti, P., Scodeggio, M., \& Martocchi, S. 2001, A\&A, 372, 29

Golev, V., \& Prugniel, P. 1998, A\&AS, 132, 255

Grebel, E. 1999, in IAU Symp. 192, The Stellar Content of Local Group Galaxies, ed. P. Whitelock \& R. Cannon (San Francisco: ASP), 17

Hinz, J., Rieke, G., \& Caldwell, N. 2003, AJ, 126, 2622

Martin, C., et al. 2005, ApJ, 619, L1

Morrissey, P., et al. 2005, ApJ, 619, L7

Neistein, E., Maoz, D., Rix, H., \& Tonry, J. 1999, AJ, 117, 2666

O'Connell, R. 1999, ARA\&A, 37, 603

Poggianti, B. 2004, in Clusters of Galaxies: Probes of Cosmological Structure and Galaxy Evolution, ed. J. S. Mulchaey, A. Dressler, \& A. Oemler (Cambridge: Cambridge Univ. Press), 246

Poggianti, B., et al. 2001, ApJ, 562, 689

Rich, R. M., et al. 2005, ApJ, 619, L107

Scodeggio, M., Gavazzi, G., Franzetti, P., Boselli, A., Zibetti, S., \& Pierini, D. 2002, A\&A, 384, 812

Trager, S. C., Faber, S. M., Worthey, G., \& González, J. J. 2000, AJ, 120, 165

Van Zee, L., Skillman, E., \& Haynes, M. 2004, AJ, 128, 121

Whitmore, B., Gilmore, D., \& Jones, C. 1993, ApJ, 407, 489

Yi, S., Demarque, P., \& Oemler, A. 1998, ApJ, 492, 480

Yi, S., et al. 2005, ApJ, 619, L111

Zibetti, S., Gavazzi, G., Scodeggio, M., Franzetti, P., \& Boselli, A. 2002, ApJ, 579,26 